\title{
Schistosoma mansoni: Expression of Fes-like tyrosine kinase SmFes in the tegument and terebratorium suggests its involvement in host penetration
}

\author{
Diana Bahia ${ }^{a, b, *}$, Renato A. Mortara ${ }^{b}$, John R. Kusel ${ }^{c}$, Luiza F. Andrade ${ }^{d}$, \\ Fernanda Ludolf ${ }^{d}$, Paula R. Kuser ${ }^{e}$, Lívia Avelar ${ }^{d}$, Jacques Trolet ${ }^{f}$, Colette Dissous ${ }^{f}$, \\ Raymond J. Pierce ${ }^{\text {f }}$, Guilherme Oliveira ${ }^{\mathrm{a}, \mathrm{d}}$ \\ a Centro de Pesquisas René Rachou (CPqRR) - FIOCRUZ, Av. Augusto de Lima 1715, Belo Horizonte, MG, 30190-002, Brazil \\ ${ }^{\mathrm{b}}$ Departamento de Microbiologia, Imunologia e Parasitologia, Escola Paulista de Medicina, UNIFESP, Rua Botucatu 862, $6^{\circ}$ andar, \\ 04023-062 São Paulo, SP, Brazil \\ ${ }^{\mathrm{c}}$ Division of Infection and Immunity, IBLS, 5th Floor, GMRC, Sir Graeme Davies Building, University Place, Glasgow University, \\ Glasgow G12 8TA, Scotland, UK \\ d Programa de Pós-Graduação e Pesquisa, Santa Casa de Belo Horizonte, Av. Francisco Sales 1111, $9^{\circ}$ andar, Ala C, Belo Horizonte, MG, 30150-221, Brazil

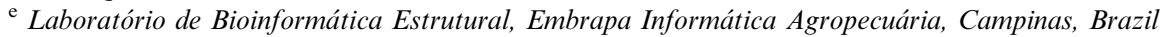 \\ ${ }^{\mathrm{f}}$ Inserm, U 547, Lille, France; Université Lille 2, Lille, France; Institut Pasteur de Lille, IFR 142, 1 rue du Professeur A. Calmette, 59019 - Lille, France
}

Received 21 April 2006; received in revised form 4 January 2007; accepted 5 January 2007

Available online 25 January 2007

\begin{abstract}
Protein Tyrosine Kinases (PTKs) are important molecules in intra- and inter-cellular communication, playing a major role in signal transduction processes. We have previously identified and characterized the molecular structure of a new PTK in Schistosoma mansoni, SmFes. SmFes exhibits the characteristic features of Fes/Fps protein tyrosine kinase subfamily of which it is the first member described in helminths. Herein, we show that genes orthologous to SmFes are also present in other Schistosoma species and the transcript is detected in Schistosoma japonicum. The SmFes protein was detected at all the main life-cycle stages and was most abundant in cercariae and newly-transformed schistosomula. However, no protein was detected in schistosomula maintained in vitro for 7 days. By immunolocalization assays we showed that SmFes is particularly concentrated at the terebratorium of miracidia and tegument of cercaria and schistosomula skin-stage. These findings suggest that SmFes may play a role in signal transduction pathways involved in larval transformation after penetration into intermediate and definitive hosts.
\end{abstract}

(C) 2007 Elsevier Inc. All rights reserved.

Index Descriptors and Abbreviations: Protein tyrosine kinase; Immunolocalization; Shistosoma, Host penetration

\section{Introduction}

Blood fluke parasites of the genus Schistosoma are the most important metazoan parasites of humans and are the

\footnotetext{
* Corresponding author. Present address: Departamento de Microbiologia, Imunologia e Parasitologia, Escola Paulista de Medicina, UNIFESP, Rua Botucatu 862, $6^{\circ}$ andar, 04023-062, São Paulo, SP, Brazil. Fax: +553132953115

E-mail address: dianabahia@hotmail.com (D. Bahia).
}

etiological agents of schistosomiasis which afflicts over 200 million people (Engels et al., 2002; http://www.who.int/ tdr/publications/publications/pdf/pr17/schisto.pdf). Transmission eradication has not been achieved and disease control relies almost solely on chemotherapy with the anthelminthic, praziquantel (Cioli and Pica-Mattoccia, 2003). Despite recent advances, especially in genomics, the goals of developing new drugs and vaccines have not yet been reached. These objectives, in addition to the understanding of parasite biology, can benefit significantly from modern 
technologies and creative use of genomics and post-genomic approaches (reviewed in Oliveira et al., 2004). We have chosen to use these approaches to target signal transduction pathways in the parasite.

The identification and characterization of signal transduction molecules and mechanisms are essential to elucidate Schistosoma host-parasite interactions and parasite biology. Like other developmental processes, the complex life-cycle of Schistosoma implies that the parasite requires permanent sensing of the environment, communication between cells of individual worms, between the worms and their hosts as well as between paired males and females, all of which involve signal transduction mechanisms. However, the molecular interactions involved in these signaling cascades are still unclear (Kapp et al., 2004; Bahia et al., 2006b).

Protein Tyrosine Kinases (PTKs) are crucial molecules in intra- and inter-cellular communication and have a major role in signal transduction processes (Hanks and Hunter, 1995). These proteins are known to be involved in development, proliferation, differentiation and communication processes of cells and hence are promising target molecules for the development of new chemotherapeutic agents, an approach intensively pursued in other areas such as cancer research (Tibes et al., 2005). There are two main classes of PTKs, receptor tyrosine kinases (RTKs) and non-receptor tyrosine kinases, also called cytoplasmic or cellular tyrosine kinases (NRTKs) (Neet and Hunter, 1996). NRTKs are known to be key players in cell proliferation and cell differentiation (Martin, 2001). Furthermore, NRTKs are involved in cell migration, adhesion and in cytoskeleton rearrangements (Brown and Cooper, 1996; Thomas and Brugge, 1997).

Among the relatively few PTKs that have been identified in Schistosoma mansoni, four are Receptor Tyrosine Kinases (RTKs). The first RTK identified is a homologue of the epidermal growth receptor SER (Shoemaker et al., 1992) characterized in adult parasite muscles. Two orthologues of the mammalian insulin receptor, SmIR1 and SmIR2 (Khayath et al., 2007; Dissous et al., 2006) are present, with conserved ligand-binding domains. A third RTK, SmRTK-1 possesses an insulin receptor-like kinase domain, but an unusual extracellular domain with a structure similar to the Venus Flytrap (VFT) module (Vicogne et al., 2003). Three NRTKs have also been identified in $S$. mansoni: TK5 is homologous to Fyn from the Src family (Kapp et al., 2001), TK4 corresponds to a Syk protein (Knobloch et al., 2002), and TK3 is a functionally active Src-like kinase (Kapp et al., 2004).

We have previously identified and characterized the molecular structure of a new Fes-like PTK in S. mansoni, SmFes (GenBank Accession No. AF515706), initially identified in the context of the EST program of Schistosome Genome Project (Oliveira et al., 2004). Although the cellular function of the Fes/Fps/Fer kinases are still largely unknown, they have been shown to be implicated in the regulation of cell-cell and cell-matrix interactions and cytoskeletal rearrangement (for review see Greer, 2002). SmFes exhibits the characteristic features of Fes/Fps protein tyrosine kinases subfamily; a coiled-coil region, $\mathrm{SH} 2$ and protein tyrosine kinase catalytic domain signatures and is the first member of Fes subfamily described in helminths.

In order to elucidate possible biological functions of SmFes in S. mansoni, we have studied the expression of the protein throughout the life-cycle and have localized SmFes by confocal immunofluorescence microscopy using a polyclonal antiserum raised against an SmFes peptide. The results give insights into possible roles of SmFes in the Schistosoma biology. In addition, a model of the last 295 amino acids of the protein corresponding to SmFes kinase domain was built by comparative protein structure modeling.

\section{Material and methods}

\subsection{Parasites}

The LE strain of $S$. mansoni has been maintained by passages through albino Biomphalaria glabrata snails (from Belo Horizonte, Minas Gerais, Brazil) at the Centro de Pesquisas René Rachou (Pellegrino and Katz, 1968). Miracidia were obtained from eggs recovered from infected livers and intestines of Swiss mice infected by a subcutaneous injection of approximately 100 cercariae (Laboratory of Malacology at the Centro de Pesquisas René Rachou (Dalton et al., 1997). Adult worms were collected by portal perfusion with Hanks-Wallace solution from mice 8 weeks post-infection and males and females were separated. Miracidia were collected, washed 2X with RPMI 1640 culture medium and fixed with $10 \%$ formaldehyde. Cercariae were collected from infected snails and maintained for $30 \mathrm{~min}$ on ice and mechanically transformed into schistosomula (Ramalho-Pinto et al., 1974). The schistosomula were then incubated in RPMI 1640 culture medium and maintained at $37^{\circ} \mathrm{C}$ for $2 \mathrm{~h}$ allowing transformation into a stage resembling skin-prepared schistosomula (Stirewalt, 1974). These schistosomula were also maintained in in vitro culture for 7 days under the conditions previously described (Harrop and Wilson, 1993).

Prior to immunolocalization assays miracidia and schistosomula were aseptically transferred to sterile tubes and pelleted by brief $(\sim 2 \mathrm{~s})$ centrifugation at $500 \mathrm{~g}$. Most of the supernatant was removed and the parasites were resuspended in $10 \%$ formaldehyde dissolved in phosphate-buffered saline $1 \times(\mathrm{PBS})$ and kept at $4{ }^{\circ} \mathrm{C}$.

\subsection{Identification of SmFes orthologues in other schistosoma species}

DNA from female and male worms was obtained by a proteinase K-based extraction method (Grevelding, 1995). The identification of SmFes in different Schistosoma species was performed using specific primers for exon 3 of the gene 
(SmFes gene organization is available on request) in a PCR assay. Primers were designed to amplify the region comprised between nucleotides 639 and 767 in the cDNA sequence (primer B: 5'-AAC ATA TAC TGA CGC GCT TGT G-3' reverse 745-767; P2for: 5'-ACG TGC ATT ATT TCA TAA A-3' forward 639-667) using FastPCR v.3.7 software (http://www.biocenter.helsinki.fi/bi/Programs/fastpcr.htm). Schistosoma haematobium, Schistosoma mattheei, Schistosoma bovis, Schistosoma curassoni, Schistosoma margrebowiei, Schistosoma intercalatum, Schistosoma japonicum, and Schistosoma rodhaini worms were kind gifts from Dr. D.A. Johnston (Natural History Museum, London, England, UK). For PCR amplification, $150 \mathrm{ng}$ of genomic DNA, $0.15 \mathrm{U}$ of Taq DNA polymerase GOLD (Stratagene), buffer (1.5 mM $\mathrm{MgCl}_{2}, 10 \mathrm{mM}$ Tris$\mathrm{HCl} \mathrm{pH} \mathrm{8.0,} \mathrm{KCl} 50 \mathrm{mM}$ ), 10 pmoles of each primer and $200 \mu \mathrm{M}$ dNTPs were mixed to a total volume of $10 \mu \mathrm{l}$. PCRs were performed with an initial denaturation for $10 \mathrm{~min}$ at $95^{\circ} \mathrm{C}$. Thirty-five cycles were run, with $1 \mathrm{~min}$ denaturation at $95^{\circ} \mathrm{C}, 1 \mathrm{~min}$ annealing at $52^{\circ} \mathrm{C}$ and $1 \mathrm{~min}$ extension at $72^{\circ} \mathrm{C}$. The final extension was for $10 \mathrm{~min}$ at $72{ }^{\circ} \mathrm{C}$. PCR products were visualized on $8 \%$ silver-stained polyacrylamide gels as previously described (Sanguinetti et al., 1994). Adult worm cDNA of S. mansoni was synthesized from adult worm total RNA, prepared using the guanidine thiocyanate/caesium chloride technique (Chirgwin et al., 1979), using the SMART RACE cDNA amplification kit (Clontech), according to the manufacturer's instructions. S. japonicum cDNA was kindly provided by Dr. Karl Hoffmann, University of Cambridge, U.K.). The cDNAs were amplified with primers ranging from nucleotides 2392-2811 (SmGOTK1: 5'-ATG CCA AAA GAC AGA TAA AAC CAT ACG-3' reverse 2811-2785 and SmGOTK2: 5'-AAT AAG CCT TTA CAT AAT CCA ACT GTC AAT G-3' forward 23922422), corresponding to exons 11-14 (unique domain of SmFes), which generated a product of $419 \mathrm{bp}$.

\subsection{Antibodies}

A synthetic peptide (Ac-SYPNTTPITFSRDPC-amide) corresponding to amino acids 583-597 of SmFes was conjugated to ovalbumin (OVA). The choice of the peptide was based on two parameters: hydrophobicity analysis of the predicted amino-acid sequence by using the ProtScale software (http://www.expasy.org/) and its localization in the poorly conserved region between the third coiled-coil domain and the SH2 domain (Fig. 4a). Anti-SmFes antibodies were produced in rabbits immunized with this peptide conjugated to OVA. The peptide, the anti-SmFes serum and pre-immune sera were all purchased from New England Peptides, Inc.

\subsection{Western blots}

Three thousand parasites (or $25 \mu \mathrm{g}$ of total protein) per sample were solubilized in Nupage LDS sample buffer
(Invitrogen), sonicated 3 times for $1 \mathrm{~min}$ and heated $10 \mathrm{~min}$ at $75^{\circ} \mathrm{C}$. Electrophoresis was performed in Nupage Bis-Tris 4-12\% gradient gels (Invitrogen) according to manufacturer's instructions and proteins were electrophoretically transferred to nitrocellulose membranes (Invitrogen). Following a blocking step with $5 \%$ powdered non-fat milk and mouse serum diluted $1 /$ 2000 in PBS, nitrocellulose sheets were incubated with anti-SmFes-1 rabbit serum (diluted 1/1000 in blocking solution) for $2 \mathrm{~h}$, followed by four washes of $5 \mathrm{~min}$ with PBS- $0.5 \%$ Tween 20 , then with horseradish peroxidase (HRP)-conjugated anti-rabbit mouse antibodies (diluted $1 / 10,000$ in blocking solution) (Sigma) for $1 \mathrm{~h}$. Bound antibodies were detected using the SuperSignal West Pico Chemiluminescent Substrate (Perbio Science) and Kodak $\mathrm{X}$-Omat films.

\subsection{Immunofluorescence studies}

Imunofluorecence assays were performed essentially according to Bahia et al. (2006c). In brief, S. mansoni forms isolated and fixed as described above (Section 2.1) were allowed to attach to poly-L-lysine coated glass slides. After a $1 \mathrm{~h}$ blocking and permeabilization step with PBS containing $0.2 \%$ gelatin (Sigma), $0.1 \%$ saponin (Sigma) and $0.1 \%$ sodium azide (PGSN), samples were incubated with antiSmFes serum diluted 1:50 in PGSN for $12 \mathrm{~h}$ in a humid chamber. After 3 washes with PBS, bound antibodies were developed with goat anti-rabbit Ig conjugated to FITC (Sigma) diluted 1:50. S. mansoni forms were then imaged by confocal microscopy as previously described (Bahia et al., 2006c).

\subsection{Comparative protein structure modeling}

Comparative or homology protein structure modeling allows the construction of a three-dimensional model for a protein of unknown structure based on a related protein of known structure (Sali and Blundell, 1993). The comparative modeling method can reliably predict the $3 \mathrm{D}$ structure of a protein with accuracy comparable to a low-resolution experimentally determined structure (Marti-Renom et al., 2002). A comparative model for the C-terminal moiety of SmFes was generated using MODELLER 7 (Sali and Blundell, 1993) based on the coordinates of the carboxyl-terminal Src kinase (Csk), a member of the Src family tyrosine kinases (PDB code id: 1k9a.pdb). The PDB database was searched using BLAST. In MODELLER, spatial and stereochemical restraints derived from the template structure and the sequence alignment were combined to form an objective function; model structures were created by optimising this function. From the alignment with the $1 \mathrm{k} 9 \mathrm{a}$ model, 10 model structures were built and evaluated with the Sting software suite (Neshich et al., 2005), and one model was used for analysis. 


\section{Results and discussion}

\subsection{SmFes homologues in other schistosome species}

Putative SmFes orthologous genes are present in at least eight other Schistosoma species (Fig. 1a), three of which are of medical importance ( $S$. japonicum, S. intercalatum and $S$. haematobium lanes 5-7, respectively) in addition to $S$. mansoni (lane 4): a 128 bp band was amplified in all species using specific primers. In addition, $S$. japonicum cDNA was shown to contain a transcript corresponding to a homologue of SmFes (Fig. 1b). However, screening S. japonicum ESTs deposited in GenBank with the peptide sequence did not allow the detection of an orthologue of SmFes. Although the detection of orthologous genes in other Schistosoma species is not surprising in itself, it suggests both a high degree of sequence conservation and the importance of this member of the Fps/Fes/Fer family in schistosomes.

\subsection{SmFes protein is differentially expressed during the parasite life cycle}

Western blots were carried out using the anti-SmFes peptide antiserum against all life-cycle stages of $S$. mansoni in order to determine the level of expression of the protein. A $\sim 143 \mathrm{kDa}$ protein was detected at all stages (Fig. 2) but cercariae showed the most marked expression (Fig. 2a). We next carried out Western blots using calibrated numbers (3000 per lane) of larvae of cercariae, mechanically prepared schistosomula after $2 \mathrm{~h}$ culture, schistosomula maintained in culture for 7 days and miracidia. This showed (Fig. 2b) that cercariae and the $2 \mathrm{~h}$ schistosomula exhibited a high level of $\mathrm{SmFes}$ protein expression, whereas this was absent in schistosomula cultured for 7 days. Expression in miracidia was weak but detectable. These data suggest that the principle role of SmFes is connected with the phase of definitive host invasion and that this protein is subsequently down-regulated. However, its expression in adults and

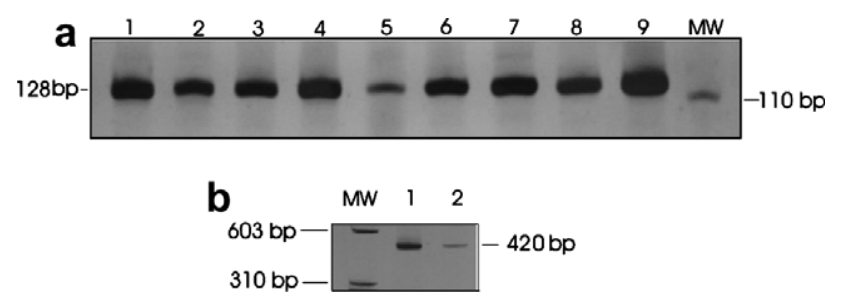

Fig. 1. (a) PCR amplification of SmFes gene orthologues in different Schistosoma species. PCR was performed using specific primers for exon 3 of the SmFes gene on DNA from: 1-S. rodhaini, 2-S. mattheei, 3-S. margrebowiei, 4-S. mansoni, 5-S. japonicum, 6-S. intercalatum, 7-S. haematobium, 8-S. curassoni and 9-S. bovis. M.W: X174 RF DNA/Hae III. (b) SmFes mRNA expression in Schistosoma japonicum. Adult worm cDNA of both $S$. mansoni and $S$. japonicum were amplified with primers ranging from bases 2392-2811 of the SmFes cDNA sequence which generated a product of $419 \mathrm{bp}$ (b, see arrow). M.W: X174 RF DNA/Hae III (Invitrogen); 1: S. mansoni, 2: S. japonicum.
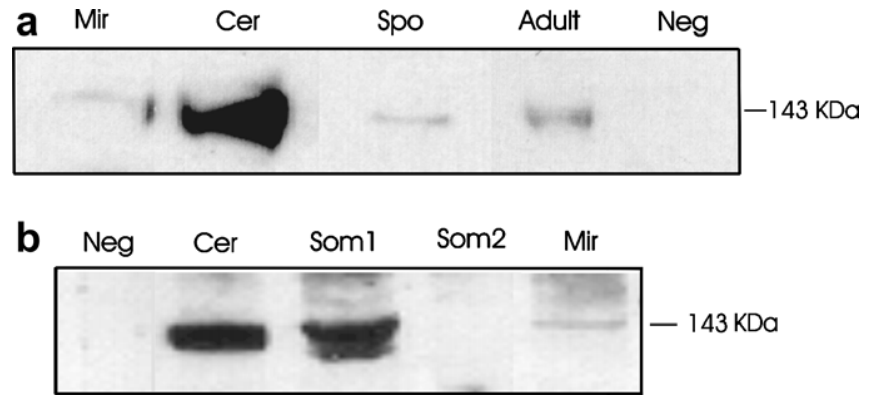

Fig. 2. SmFes protein expression in different stages of the Schistosoma mansoni life cycle. (a) Western blot of proteins extracts $(25 \mu \mathrm{g})$ of various life cycle stages of $S$. mansoni using the anti-SmFes peptide antiserum. The lanes represented are protein extracts of miracidia (Mir), cercariae (Cer), primary sporocysts (Spo), mixed adult worms (Adult) and no extract (Neg). (b) Western blot of larval stages (3000 larvae per lane) showing the extinction of the signal in 7 day old cultured schistosomula (Som2) compared to cercariae (Cer) and $2 \mathrm{~h}$ mechanically-prepared schistosomula (Som1) as well as a negative control (Neg). Pre-immune sera (not shown) were always negative.

miracidia also indicates that it has other roles during the life-cycle, consistent with the function of members of the Fps/Fes/Fer family in other organisms (Greer, 2002).

\subsection{Localization of SmFes in cercariae, schistosomula and miracidia}

In order to determine whether the high level of protein expression detected by Western blotting in cercariae and schistosomula correlated with a particular localization of the protein, we carried out immunolocalization studies targeted on the larval stages (Fig. 3). These experiments show that $\mathrm{SmFes}$ is present in the pharyngeal musculature of the cercaria (Fig. 3B, arrow 1) and also in the tegument of both the cercaria (Fig. 3B, arrow 2) and schistosomulum (Fig. 3D, arrows 3).

The profound environmental changes experienced by the parasite as it passes from fresh water to the skin and tissues of the mammalian host are accompanied by secretions from the acetabular glands, and the reorganization of parasite tissues following these secretory processes (Hockley and McLaren, 1973; Stirewalt, 1974). There are also surface membrane and cytoskeletal changes (Skelly and Shoemaker, 2001) and there is rapid repair of the lesion formed after the loss of the tail of the cercaria, just after skin penetration (Stirewalt, 1974). These changes and the expression of SmFes at the surface of cercariae and newlytransformed schistosomula are consistent with the known function of mammalian orthologues of SmFes in regulating cytoskeletal rearrangements (Greer, 2002).

Tissue remodeling and perhaps the repair processes are likely to be carried out by the lysosomal/autophagosome system. This was first described for helminthes by Threadgold and Arme (1974) and in gastric epithelial cells by Bogitsch (1975). In mammalian cells and yeast, the lysosome and autophagosome pathway is induced by signals acting on phosphatidylinositol 3-kinase (PI3 kinase; see Engelman 

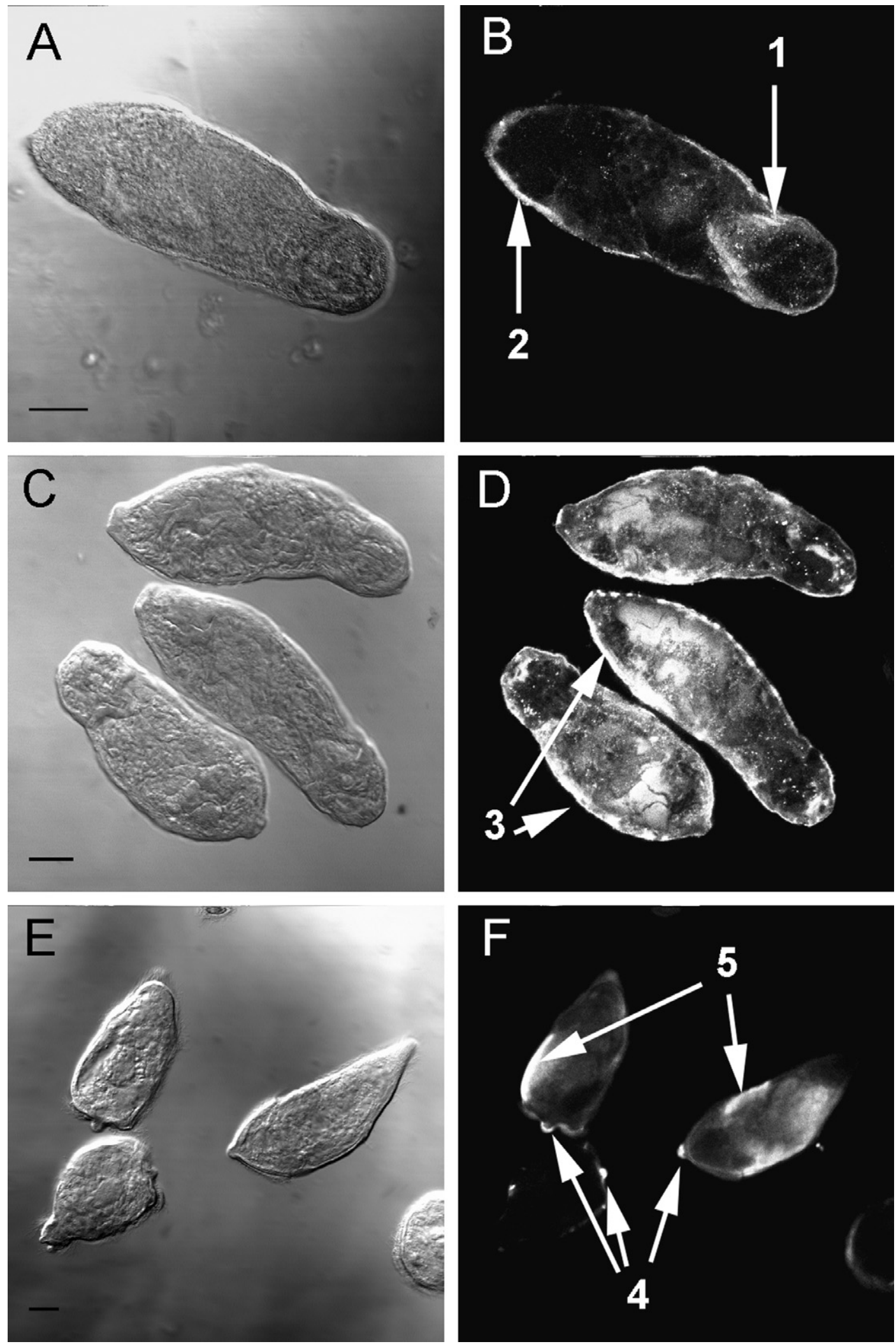

Fig. 3. Immunolocalization of SmFes in Miracidia, Cercariae and Schistosomula skin-stage. Differential interference contrast and corresponding fluorescence confocal microscopy images of cercariae (A and B), shistosomula (C and D) and miracidia (E and F) stages. Anti-S. mansoni SmFes antibody (1:50) and secondary antibody conjugated to FITC (SIGMA) were used to detect SmFes. SmFes localized to the pharyngeal musculature of the cercaria (1) and also in the tegument of both the cercariae, (2) schistosomulum (3), and to the terebratorium (4) and the tegument (5) of miracidia. Negative controls carried out with pre-immune serum (1:50) showed no fluorescence (not shown). Magnification bars $=20 \mu \mathrm{m}$. 
et al., 2006 for review). This pathway occurs in the schistosomulum as was shown by inhibitors of the PI3 kinase stimulated vesicular pathway, such as wortmannin, 3-methyl adenine, and $\mathrm{N}$-ethylmaleimide (Al-Adhami et al., 2005). Interestingly, Fes has been shown to interact functionally with complexes containing PI3 kinase and the insulin receptor substrate (IRS) (Iwanishi et al., 2000). If this is also the case in schistosomes, SmFes would be involved in the membrane transformations leading to metabolic changes including the increase in glucose transport during schistosomulum development. This would also be consistent with the molecular events involved in this process elegantly described by Skelly and Shoemaker (2001), in which the glucose transporter SGTP4 moves from intracellular vesicles into the surface membrane by membrane fusion. Finally, this also coincides with the localization of the insulin receptor orthologue SmIR1 in the basal tegumental membrane (Khayath et al., 2007).

Lysosomes and autophagosomes were not found in cercariae or miracidia, but formed rapidly during transformation of the cercariae into schistosomula (Al-Adhami et al., 2005) or the miracidia into mother sporocysts (Ana de Mattos, personal communication).

\subsection{The role of the miracidial terebratorium}

In miracidia (Fig. 3E and F), SmFes is expressed mainly in the terebratorium (Fig. 3F, arrows 4 ) and the tegument (Fig. 3B, arrows 5). The terebratorium (Reisinger, 1923) is frequently referred to as the anterior or apical papilla by many authors. It is a hemispheric structure and consists of a number of membranous folds, equipped with abundant sensory terminals (Ebrahimzadeh, 1977; Pan, 1980, 1996). This terebratorium helps the miracidium in the attachment to its snail host immediately prior to penetration. This is preceded by fixation of the larva by the terebratorium (acting as a sucker) and secretions from the apical gland cells. Upon penetration the ciliated epidermal plates are shed and the longitudinal muscle layers disappear as the miracidium develops into the primary sporocyst in the snail intermediate host (Bahia et al., 2006a).

Overall, our results show that SmFes is expressed both at the terebratorium of miracidia and tegument of skinstage schistosomula ( $2 \mathrm{~h}$ after transformation of cercariae into schistosomula) and we can speculate that a protein kinase in this location might be intimately involved in signaling processes during the penetration of the miracidium and in the transformation process of both cercariae and miracidia.

We are currently engaged in a study using pull-down assays coupled to proteomics and two hybrid systems, two-dimensional gel electrophoresis, mass spectrometry and biochemical assays aiming to search for partners interacting with SmFes. We are also investigating the potential interaction of SmFes with the PI3 kinase isoform involved in insulin pathway signalling (PI3 kinase 1A; see Engelman et al., 2006 for review). These approaches will provide evi- dence of the signal transduction pathways in which SmFes is involved in order to understand the molecular processes during the development of schistosomes and deepen the current knowledge on host-parasite interactions.

\subsection{Modeling of the SmFes protein}

The SmFes protein has an overall conserved domain structure compared to other members of the Fes/Fps family (Fig. 4a). The $\mathrm{N}$-terminal region contains 3 putative coiledcoil domains involved in oligomerization of the protein and possibly in interactions with other coiled-coil domain proteins such as p120catenin (Greer, 2002). The SH2 domain precedes the catalytic domain and is involved in proteinprotein interactions via the recognition of phosphotyrosine residues on partner proteins such as the EGF receptor or
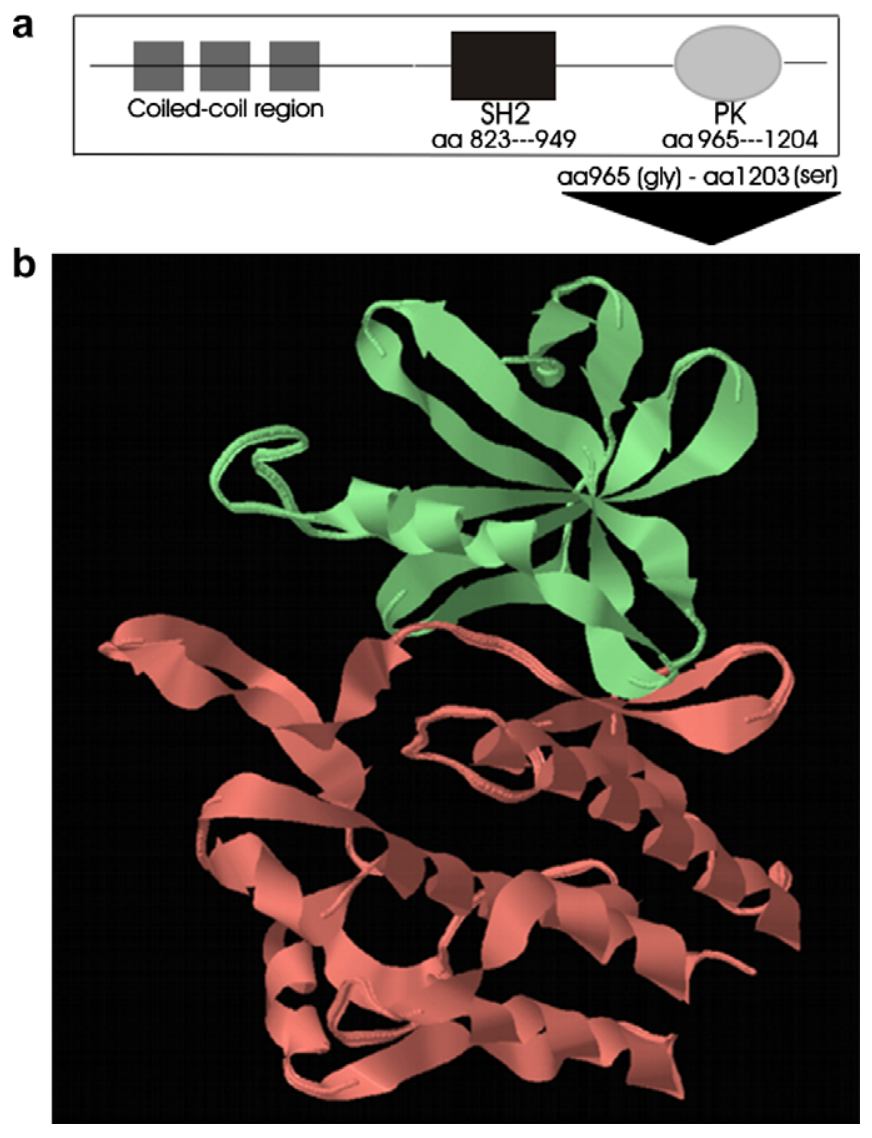

Fig. 4. SmFes modeling and protein structure. (a) Schematic diagram of SmFes protein structure. SmFes exhibits the characteristic features of Fes/ Fps protein tyrosine kinases subfamily, an $\mathrm{SH} 2$ and a protein tyrosine kinase catalytic domain signature. The unique domain in $\mathrm{SmFes}$ is localized before the SH2 signature and contains three coiled-coil regions. (b) Theoretical 3-dimensional model of the of Schistosoma mansoni Feslike tyrosine kinase $\mathrm{C}$-terminal region comprised between residues 965 and 1203 , obtained by comparative modeling with the kinase domain of $1 \mathrm{k} 9 \mathrm{a}$. The light green portion corresponds to the $\mathrm{N}$-domain of the kinase structure, and the salmon portion corresponds to the C-domain. The black downward arrowhead indicates the region modeled in the primary SmFes protein structure. (For interpretation of the references to color in this figure legend, the reader is referred to the web version of this paper.) 
PI3 kinase (Greer, 2002). The catalytic domain is well-conserved and a model of the C-terminal moiety of the SmFes protein, containing this domain, was generated (Fig. 4b). The modeled stretch corresponds to the region comprised between residues 965 (Gln) and 1203 (Asn) of full length SmFes. Sequence alignments (not shown) with the database of structures revealed that this region has $41 \%$ identity and $62 \%$ of conserved residues with the $\mathrm{N}$ - and C-lobes (amino acids 191-431) of the kinase domain of carboxyl-terminal Src kinase (PDB id: 1k9a; Ogawa et al., 2002). The high level of similarity justified its use as a template for modeling part of SmFes. The alignment was used to generate a homology model (Fig. 4b). As expected from the building procedure, the model resembles that of the kinase domain of the $1 \mathrm{k} 9 \mathrm{a}$ structure ( $\mathrm{SH} 2$ and $\mathrm{SH} 3$ domains removed). The overall structure is formed by an $\alpha \beta$ domain composed of $5 \beta$-strands and an $\alpha$-helix and a mainly $\alpha$ domain with eight $\alpha$-helices (number of strands and helices are according to the topology calculated by PDBSum (Laskowski, 2001). The obtained model shows good stereochemistry, as checked by the Ramachandran Diagram (not shown). Two regions corresponding to the functional sites described by Prosite (Hulo et al., 2006); "protein kinase ATP-binding region signature" and "tyrosine protein kinases specific active-site signature" are present in this structure. The protein kinase ATP-binding region formed by 22 amino acids is located in the three initial $\beta$ strands of the structure which are structurally conserved.

The protein tyrosine kinase region is located in a loop connecting the largest $\alpha$-helix in the structure and a small $\beta$-strand. Considering that nine out of the thirteen residues that characterize this signature are conserved, and the loop is situated in the interior of the protein, it is expected that the structure maintains its similarity to the model. Among the four amino acid substitutions, two residues are changed to cysteine. However, these new cysteine residues do not form a disulfide bridge in the structure. Within this stretch of amino acids the catalytic residues Asp132 (Asp314 in 1k9a), Ala134 (Ala316 in $1 \mathrm{k} 9 \mathrm{a}), \operatorname{Arg} 136(\mathrm{Arg} 318$ in $1 \mathrm{k} 9 \mathrm{a})$ classified according to the Catalytic Site Atlas (Porter et al., 2004) are conserved. Not only are these amino acids conserved, but also all the interactions that they perform in the modelled structure, like the charged-charged interaction between $A s p(132 / 314)$ and $\operatorname{Arg}(136 / 318)$, and particularly those allowing the recognition and positioning of the substrate are maintained.

This conserved overall structure, particularly concerning the ATP-binding site in the N-lobe (not shown) suggests that the development of schistosome-specific inhibitors targeting this site and the catalytic domain in general will be difficult. Further efforts to model the amino acids located just before the SmFes kinase domain, and particularly the less well-conserved $\mathrm{SH} 2$ domain, will hopefully provide better targets for the design of new anti-schistosoma drugs.

\section{Acknowledgments}

The work was supported by the Inserm-Fiocruz collaborative programme, the Institut National de la Santé et de la Recherche Médicale (U547), the Institut Pasteur de Lille, the Centre National de la Recherche Scientifique and the Microbiology program of the Ministère de l'Education Nationale, de la Recherche et de la Technologie (MENRT). The financial support from FIOCRUZ/PDTIS (GO), PAPES (GO 400315/2006-8) and FAPEMIG (DB CBB-174/02) is also acknowledged. G.O., R.A.M., and D.B. are $\mathrm{CNPq}$ fellows.

We thank Dr. K. Hoffman (University of Cambridge, U.K.) for providing $S$. japonicum cDNA and Dr. D.A. Johnston (Natural History Museum, London, U.K.) for providing DNA from various schistosome species.

\section{References}

Al-Adhami, B.H., Noble, C., Sharaf, O., Thornhill, J., Doenhoff, M.J., Kusel, J.R., 2005. The role of acidic organelles in the development of schistosomula of Schistosoma mansoni and their response to signalling molecules. Parasitology 130, 309-322.

Bahia, D., Avelar, L.G.A., Vigorosi, F., Cioli, D., Oliveira, G.C., Mortara, R.A., 2006a. The distribution of motor proteins in the muscles and flame cells of the Schistosoma mansoni miracidium and primary sporocyst. Parasitology 133, 321-329.

Bahia, D., Freire, L., Ludolf, F., Mortara, R.A., Oliveira, G.C., $2006 \mathrm{~b}$. Protein tyrosine kinases in Schistosoma mansoni. Memórias do Instituto Oswaldo Cruz 101, 137-143.

Bahia, D., Avelar, L., Mortara, R.A., Khayath, N., Yan, Y., Noel, C., Capron, M., Dissous, C., Pierce, R.J., Oliveira, G.C., 2006c. SmPKC1, a new protein kinase $\mathrm{C}$ identified in the platyhelminth parasite Schistosoma mansoni. Biochemical and Biophysical Research Communications $345,1138-1148$.

Bogitsch, B.J., 1975. Cytochemistry of gastrodermal autophagy following starvation in Schistosoma mansoni. Journal of Parasitology 61, 237-248.

Brown, M.T., Cooper, J.A., 1996. Regulation, substrates and functions of src. Biochimica et Biophysica Acta 1287, 121-149.

Chirgwin, J.M., Przybyla, A.E., MacDonald, R.J., Rutter, W.J., 1979. Isolation of biologically active ribonucleic acid from sources enriched in ribonuclease. Biochemistry 18, 5294-5299.

Cioli, D., Pica-Mattoccia, L., 2003. Praziquantel Parasitology Research 90 (Supp. 1), S3-S9.

Dalton, J.P., Day, S.R., Drew, A.C., Brindley, P.J., 1997. A method for the isolation of schistosome eggs and miracidia free of contaminating host tissues. Parasitology 115, 29-32.

Dissous, C., Khayath, N., Vicogne, J., Capron, M., 2006. Growth factor receptors in helminth parasites: signalling and host-parasite relationships. FEBS Letters 580, 2968-2975.

Ebrahimzadeh, A., 1977. Contributions to the micromorphology of the miracidium of Schistosoma mansoni. I Fine structure of the tegument and its "associated structures" Zeitschrift Für Parasitenkunde 54, 257-267.

Engelman, J.A., Luo, J., Cantley, L.C., 2006. The evolution of phosphatidylinositol 3-kinases as regulators of growth and metabolism. Nature Reviews Genetics 7, 606-619.

Engels, D., Chitsulo, L., Montresor, A., Savioli, L., 2002. The global epidemiological situation of schistosomiasis and new approaches to control and research. Acta Tropica 82, 139-146.

Greer, P., 2002. Closing in on the biological functions of Fps/Fes and Fer. Nature Reviews Molecular Cell Biology 3, 278-289. 
Grevelding, C.G., 1995. The female-specific W1 sequence of the Puerto Rican strain of Schistosoma mansoni occurs in both genders of a Liberian strain. Molecular and Biochemical Parasitology 71, 269-272.

Hanks, S.K., Hunter, T., 1995. Protein kinases 6. The eukaryotic protein kinase superfamily: kinase (catalytic) domain structure and classification. The FASEB Journal: Official Publication of the Federation of American Societies for Experimental Biology 9, 576-596.

Harrop, R., Wilson, R.A., 1993. Protein synthesis and release by cultured schistosomula of Schistosoma mansoni. Parasitology 107 (Pt 3), 265-274.

Hockley, D.J., McLaren, D.J., 1973. Schistosoma mansoni: changes in the outer membrane of the tegument during development from cercaria to adult worm. International Journal of Parasitology 3, 13-25.

Hulo, N., Bairoch, A., Bulliard, V., Cerutti, L., De Castro, E., Langendijk-Genevaux, P.S., Pagni, M., Sigrist, C.J.A., 2006. The PROSITE database. Nucleic Acids Research 34, D227-D230.

Iwanishi, M., Czech, M.P., Cherniack, A.D., 2000. The protein tyrosine kinase Fer associates with signaling complexes containing Insulin receptor substrate-1 and phosphatidylinositol 3-kinase. Journal of Biological Chemistry 275, 38995-39000.

Kapp, K., Knobloch, J., Schussler, P., Sroka, S., Lammers, R., Kunz, W., Grevelding, C.G., 2004. The Schistosoma mansoni Src kinase TK3 is expressed in the gonads and likely involved in cytoskeletal organization. Molecular and Biochemical Parasitology 138, 171-182.

Kapp, K., Schussler, P., Kunz, W., Grevelding, C.G., 2001. Identification, isolation and characterization of a Fyn-like tyrosine kinase from Schistosoma mansoni. Parasitology 122, 317-327.

Khayath, N., Vicogne, J., Ahier, A., Ben Younes, A., Konrad, C., Trolet, J., Viscogliosi, E., Brehm, K., Dissous, C., 2007. Diversification of the insulin receptor family in the helminth parasite Schistosoma mansoni. FEBS Journal 274, 659-676.

Knobloch, J., Winnen, R., Quack, M., Kunz, W., Grevelding, C.G., 2002. A novel Syk-family tyrosine kinase from Schistosoma mansoni which is preferentially transcribed in reproductive organs. Gene 294, 87-97.

Laskowski, R.A., 2001. PDBsum: summaries and analyses of PDB structures. Nucleic Acids Research 29, 221-222.

Martin, G.S., 2001. The hunting of the Src. Nature Reviews Molecular Cell Biology 2, 467-475.

Marti-Renom, M.A., Madhusudhan, M.S., Fiser, A., Rost, B., Sali, A., 2002. Reliability of assessment of protein structure prediction methods. Structure 10, 435-440.

Neet, K., Hunter, T., 1996. Vertebrate non-receptor protein-tyrosine kinase families. Genes to Cells: Devoted to Molecular \& Cellular Mechanisms 1, 147-169.

Neshich, G., Borro, L.C., Higa, R.H., Kuser, P.R., Yamagishi, M.E., Franco, E.H., Krauchenco, J.N., Fileto, R., Ribeiro, A.A., Bezerra, G.B., Velludo, T.M., Jimenez, T.S., Furukawa, N., Teshima, H., Kitajima, K., Bava, A., Sarai, A., Togawa, R.C., Mancini, A.L., 2005. The diamond STING server. Nucleic Acids Research 33, 29-35.

Ogawa, A., Takayama, Y., Sakai, H., Chong, K.T., Takeuchi, S., Nakagawa, A., Nada, S., Okada, M., Tsukihara, T., 2002. Structure of the carboxyl-terminal Src kinase, Csk. Journal of Biological Chemistry 277, 14351-14354.

Oliveira, G., Rodrigues, N.B., Romanha, A.J., Bahia, D., 2004. Genome and genomics of schistosomes. Canadian Journal of Zoology-Revue Canadienne de Zoologie 82, 375-390.

Pan, S.C., 1980. The fine structure of the miracidium of Schistosoma mansoni. Journal of Invertebrate Pathology 36, 307-372.

Pan, S.C., 1996. Schistosoma mansoni: the ultrastructure of larval morphogenesis in Biomphalaria glabrata and of associated hostparasite interactions. Japanese Journal of Medical Science and Biology 49, 129-149.

Pellegrino, J., Katz, N., 1968. Experimental chemotherapy of Schistosomiasis mansoni. Advances in Parasitology 6, 233-290.

Porter, C.T., Bartlett, G.J., Thornton, J.M., 2004. The catalytic site atlas: a resource of catalytic sites and residues identified in enzymes using structural data. Nucleic Acids Research 32, D129-D133.

Ramalho-Pinto, F.J., Gazzinelli, G., Howells, R.E., Mota-Santos, T.A., Figueiredo, E.A., Pellegrino, J., 1974. Schistosoma mansoni: defined system for stepwise transformation of cercaria to schistosomule in vitro. Experimental Parasitology 36, 360-372.

Reisinger, E., 1923. Studies on the structure and function of the excretory apparatus of digenetic trematodes I. Emunctory of the miracidia of Schistosoma haematobium Bilharz together with studies on their anatomy and histology. Zoologische Anzeiger (Leipzig) 57, 1-20.

Sali, A., Blundell, T.L., 1993. Comparative protein modelling by satisfaction of spatial restraints. Journal of Molecular Biology 234, $779-815$.

Sanguinetti, C.J., Dias, N.E., Simpson, A.J., 1994. Rapid silver staining and recovery of PCR products separated on polyacrylamide gels. BioTechniques 17, 914-921.

Shoemaker, C.B., Ramachandran, H., Landa, A., dos Reis, M.G., Stein, L.D., 1992. Alternative splicing of the Schistosoma mansoni gene encoding a homologue of epidermal growth factor receptor. Molecular and Biochemical Parasitology 53, 17-32.

Skelly, P., Shoemaker, C., 2001. The Schistosoma mansoni host-interactive tegument forms from vesicle irruptions of a cyton network. Parasitology $122,67-73$.

Stirewalt, M.A., 1974. Schistosoma: Cercaria to schistosomule. Advances in Parasitology 12, 115-182.

Thomas, S.M., Brugge, J.S., 1997. Cellular functions regulated by Src family kinases. Annual Review of Cell and Developmental Biology 13, 513-609.

Tibes, R., Trent, J., Kurzrock, R., 2005. Tyrosine kinase inhibitors and the dawn of molecular cancer therapeutics. Annual Review of Pharmacology and Toxicology 45, 357-384.

Threadgold, L.T., Arme, C., 1974. Electron microscope studies of Fasciola hepatica XI: autophagy and parenchymal cell function. Experimental Parasitology 35, 389-405.

Vicogne, J., Pin, J.P., Lardans, V., Capron, M., Noel, C., Dissous, C., 2003. An unusual receptor tyrosine kinase of Schistosoma mansoni contains a Venus Flytrap module. Molecular and Biochemical Parasitology 126, 51-62. 